\title{
Fredholm theory of Toeplitz operators on doubling Fock Hilbert spaces
}

Article

Accepted Version

Al-Qabani, A., Hilberdink, T. and Virtanen, J. A. (2020)

Fredholm theory of Toeplitz operators on doubling Fock Hilbert spaces. Mathematica Scandinavica, 126 (3). pp. 593-602.

ISSN 1903-1807 doi: https://doi.org/10.7146/math.scand.a120920 Available at https://centaur.reading.ac.uk/85955/

It is advisable to refer to the publisher's version if you intend to cite from the work. See Guidance on citing.

Published version at: https://www.mscand.dk/forthcoming

To link to this article DOI: http://dx.doi.org/10.7146/math.scand.a-120920

Publisher: Mathematica Scandinavica

All outputs in CentAUR are protected by Intellectual Property Rights law, including copyright law. Copyright and IPR is retained by the creators or other copyright holders. Terms and conditions for use of this material are defined in the End User Agreement.

\section{www.reading.ac.uk/centaur}

\section{CentAUR}

Central Archive at the University of Reading

Reading's research outputs online 


\title{
FREDHOLM THEORY OF TOEPLITZ OPERATORS ON DOUBLING FOCK HILBERT SPACES
}

\author{
AAMENA AL-QABANI, TITUS HILBERDINK AND JANI A. VIRTANEN
}

\begin{abstract}
We study the Fredholm properties of Toeplitz operators acting on doubling Fock Hilbert spaces, and describe their essential spectra for bounded symbols of vanishing oscillation. We also compute the index of these Toeplitz operators in the special case when $\varphi(z)=|z|^{\beta}$ with $\beta>0$. Our work extends the recent results on Toeplitz operators on the standard weighted Fock spaces to the setting of doubling Fock spaces.
\end{abstract}

\section{INTRODUCTION}

A positive Borel measure $\mu$ on the complex plane $\mathbb{C}$ is said to be a doubling measure if there is a positive constant $C$ such that

$$
\mu(D(z, 2 r)) \leq C \mu(D(z, r))
$$

for all $z \in \mathbb{C}$ and $r>0$, where

$$
D(z, r)=\{w \in \mathbb{C}:|w-z|<r\} .
$$

We denote by $d A$ the standard Lebesgue area measure on $\mathbb{C}$. Let $\varphi$ be a subharmonic nonharmonic real-valued function of class $C^{2}$ on the complex plane $\mathbb{C}$ such that $\Delta \varphi d A$ is a doubling measure, where $\Delta \varphi$ is the Laplacian of the function $\varphi$ defined by

$$
\Delta \varphi=\varphi_{x x}+\varphi_{y y} .
$$

The doubling Fock space $F_{\varphi}^{2}$ is defined by

$$
F_{\varphi}^{2}=\left\{f \in H(\mathbb{C}):\|f\|_{\varphi}^{2}=\int_{\mathbb{C}}|f(z)|^{2} e^{-2 \varphi(z)} d A(z)<\infty\right\},
$$

where $H(\mathbb{C})$ is the set of all entire functions. These spaces were introduced in [6], where their sampling and interpolating sequences were described.

It is well known that $F_{\varphi}^{2}$ is a Hilbert space with inner product given by

$$
\langle f, g\rangle=\int_{\mathbb{C}} f(w) \overline{g(w)} e^{-2 \varphi(w)} d A(w) .
$$

We also note that the doubling Fock spaces include the standard weighted Fock spaces [14], the Fock-Sobolev space [3], the Fock spaces with weights $\varphi(z)=|z|^{\beta}$, where $\beta>0$ (see [10]), and the generalized Fock spaces [11] with weights $\varphi$ satisfying $0<C_{1} \leq \Delta \varphi(z) \leq C_{2}$, for all $z \in \mathbb{C}$, where $C_{1}$ and $C_{2}$ are positive constants.

2010 Mathematics Subject Classification. Primary 47B35; Secondary 30H20.

Key words and phrases. Toeplitz operators, Hankel operators, doubling Fock spaces, Fredholm properties, essential spectrum.

J. Virtanen was supported in part by Engineering and Physical Sciences Research Council grant $\mathrm{EP} / \mathrm{M} 024784 / 1$. 
When $\Delta \varphi d A$ is doubling, we say that a measurable function $f: \mathbb{C} \rightarrow \mathbb{C}$ is in $L_{\varphi}^{2}$ if

$$
\|f\|_{\varphi}^{2}=\int_{\mathbb{C}}|f(z)|^{2} e^{-2 \varphi(z)} d A(z)<\infty .
$$

We denote by $P$ the orthogonal projection of $L_{\varphi}^{2}$ onto $F_{\varphi}^{2}$. It has the integral representation

$$
P f(z)=\int_{\mathbb{C}} f(w) \overline{K_{z}(w)} e^{-2 \varphi(w)} d A(w),
$$

where $K_{z}$ is the reproducing kernel function; that is, for each $z \in \mathbb{C}, K_{z}$ is the unique function in $F_{\varphi}^{2}$ for which

$$
f(z)=\left\langle f, K_{z}\right\rangle
$$

for all $f \in F_{\varphi}^{2}$. Note that $K_{z}$ depends on $\varphi$ and we write

$$
K_{z}(w)=K_{\varphi}(w, z)=K(w, z),
$$

for $z, w \in \mathbb{C}$. For $z \in \mathbb{C}$, the normalized reproducing kernel function at $z$ is defined by

$$
k_{z}(w)=\frac{K_{\varphi}(w, z)}{\left\|K_{\varphi}(z, z)\right\|_{\varphi}},
$$

for $w \in \mathbb{C}$.

For $f \in L^{\infty}=L^{\infty}(\mathbb{C})$, the Toeplitz operator $T_{f}$ on $F_{\varphi}^{2}$ with symbol $f$ is defined by

$$
T_{f}(g)=P(f g) \text {. }
$$

It is clearly a bounded linear operator on $F_{\varphi}^{2}$ and $\left\|T_{f}\right\| \leq\|f\|_{\infty}$.

We say that a bounded linear operator $T$ on a Banach space $X$ is Fredholm if $\operatorname{ker} T$ and $X / T(X)$ are both finite-dimensional. In this case, the index of $T$ is defined to be

$$
\text { ind } T=\operatorname{dim} \operatorname{ker} T-\operatorname{dim}(X / T(X)) \text {. }
$$

The essential spectrum $\sigma_{\text {ess }}(T)$ is defined by

$$
\sigma_{\text {ess }}(T)=\{\lambda \in \mathbb{C}: T-\lambda I \text { is not Fredholm }\},
$$

where $I$ denotes the identity operator on $X$. Clearly $\sigma_{\text {ess }}(T)$ is contained in the spectrum $\sigma(T)$ of $T$.

Our main results are concerned with the Fredholm properties and computation of the index of Toeplitz operators acting on doubling Fock spaces $F_{\varphi}^{2}$ with bounded symbols of vanishing oscillation. Before introducing the notion of vanishing oscillation, we make a few useful observations. Since $d \mu=\Delta \varphi d A$ is a doubling measure, by [12, §I.8.6], for any $z \in \mathbb{C}$ and $r>0$, we have that

$$
\mu(\partial(D(z, r))=\mu(\{z\})=0
$$

and $\mu(D(z, 2 r)) \geq C \mu(D(z, r))$, where $C>1$ is a constant. Moreover, our assumption that $\varphi$ is non-harmonic implies that $\mu$ is a locally finite non-zero doubling measure on $\mathbb{C}$, so $0<\mu(D(z, r))<\infty$, for any $z \in \mathbb{C}$ and $r>0$. It follows that, for each $z \in \mathbb{C}, \mu(D(z, r)) \rightarrow$ $\infty$ as $r \rightarrow \infty$, and the function $r \mapsto \mu(D(z, r))$ is an increasing homeomorphism from the interval $(0, \infty)$ onto itself. Hence, for every $z \in \mathbb{C}$, there is a unique radius $\rho(z)$ such that

$$
\mu(D(z, \rho(z))=1 .
$$


For $z \in \mathbb{C}$ and $r>0$, we denote by $D^{r}(z)$ the disk $D(z, r \rho(z))$. Then we say that $f$ is of vanishing oscillation and write $f \in V O$ if $f$ is a continuous function on $\mathbb{C}$ and

$$
\omega_{r}(f)(z)=\sup _{w \in D^{r}(z)}|f(z)-f(w)| \rightarrow 0
$$

as $z \rightarrow \infty$. Note that $V O$ is independent of the choice of $r$.

\section{MAin RESUlts}

The Fredholm properties of Toeplitz operators on the unweighted Fock space $F^{2}$ were described by Berger and Coburn [2] in 1987 using heavy machinery of $C^{*}$-algebras suitable for operators on Hilbert spaces. In 1992, Stroethoff [13] provided elementary proofs of their results, and very recently the theory was extended to the standard weighted Fock spaces $F_{\alpha}^{p}$ using elementary methods [1] and limit operator techniques [4]. In the following theorem, we extend the theory to doubling Fock Hilbert spaces $F_{\varphi}^{2}$, which also sets the stage for further extensions to other doubling Fock spaces $F_{\varphi}^{p}$.

Theorem 1. Let $f \in L^{\infty} \cap V O$. Then the Toeplitz operator $T_{f}$ on $F_{\varphi}^{2}$ is Fredholm if and only if there is a radius $R>0$ such that $f$ is bounded away from zero on $\mathbb{C} \backslash D(0, R)$, that $i s, \inf _{|z| \geq R}|f(z)|>0$. In this case,

$$
\sigma_{\mathrm{ess}}\left(T_{f}\right)=\bigcap_{R>0} \operatorname{cl} f(\mathbb{C} \backslash D(0, R)),
$$

where cl stands for the closure of the given set.

The difficulty with computing the index of Toeplitz operators on $F_{\varphi}^{2}$ is that doubling Fock spaces do not have simple bases unlike the standard weighted Fock spaces where index formulas can be obtained more easily; see [2] for Toeplitz operators on $F^{2}$ and [1] for these operators on $F_{\alpha}^{p}$. The following result gives an index formula in the special case $\varphi(z)=\frac{1}{2}|z|^{\beta}$ with $\beta>0$, which was introduced in [9] to study the properties of Hankel operators. For convenience, we write $F_{|z|^{\beta}}^{2}$ for $F_{\frac{1}{2}|z|^{\beta}}^{2}$. It is not difficult to see that $F_{|z|^{\beta}}^{2}$ is a doubling Fock space (and not a standard weighted Fock space).

Theorem 2. Let $f \in L^{\infty} \cap V O$. Then the Toeplitz operator $T_{f}$ on $F_{|z|^{\beta}}^{2}$ is Fredholm if and only if there is a radius $R>0$ such that $f$ is bounded away from zero on $\mathbb{C} \backslash D(0, R)$. In this case,

$$
\operatorname{ind}\left(T_{f}\right)=-\operatorname{wind}\left(\left.f\right|_{\{|z|=R\}}\right) \text {, }
$$

where wind $\left(\left.f\right|_{\{|z|=R\}}\right)$ is the winding number of the curve $f(\{|z|=R\})$ around the origin.

The proofs of our main theorems are given in Section 4 below.

\section{Preliminaries}

The following estimate for the Bergman kernel for $F_{\varphi}^{2}$ plays an important role in the study of concrete operators (such as Toeplitz and Hankel operators) on doubling Fock spaces (see [5, 8]). 
Theorem 3 ([7, Theorem 1.1 and (3)]). There are constants $C>0$ and $\epsilon>0$ such that

$$
\left|K\left(z_{1}, z_{2}\right)\right| \leq C \frac{1}{\rho\left(z_{1}\right) \rho\left(z_{2}\right)} \frac{e^{\varphi\left(z_{1}\right)+\varphi\left(z_{2}\right)}}{\exp \left(d_{\varphi}\left(z_{1}, z_{2}\right)^{\epsilon}\right)},
$$

for any $z_{1}, z_{2} \in \mathbb{C}$, where $d_{\varphi}$ is the distance on $\mathbb{C}$ induced by the metric $\varphi(z)^{-2} d z \otimes d \bar{z}$.

The previous theorem combined with the estimate (see [7, Proposition 2.10])

$$
C^{-1} \frac{e^{\varphi(z)}}{\rho(z)} \leq\left\|K_{\varphi}(\cdot, z)\right\|_{\varphi} \leq C \frac{e^{\varphi(z)}}{\rho(z)}, \quad \text { for } z \in \mathbb{C},
$$

where $C$ is a positive constant, gives the following result.

Lemma 4 ([8, Lemma 2.7]). Let $r \geq 1$. For every $k \geq 0$ and $\epsilon>0$ there exists a constant $C_{k, \epsilon}(r)>0$ such that

$$
\int_{\mathbb{C} \backslash D^{r}\left(z_{2}\right)} \frac{\left|z_{1}-z_{2}\right|^{k}}{\exp \left(d_{\varphi}\left(z_{1}, z_{2}\right)^{\epsilon}\right)} \frac{d A\left(z_{1}\right)}{\rho\left(z_{1}\right)^{2}} \leq C_{k, \epsilon}(r) \rho\left(z_{2}\right)^{k}, \quad \text { for any } z_{2} \in \mathbb{C} .
$$

Moreover, $C_{k, \epsilon}(r) \rightarrow 0$ as $r \rightarrow \infty$, for every $k \geq 0$ and $\epsilon>0$.

For $f \in L^{\infty}$, the Berezin transform $\tilde{f}$ of $f$ is defined by

$$
\tilde{f}(z)=\left\langle f k_{z}, k_{z}\right\rangle=\int_{\mathbb{C}}\left|k_{z}(w)\right|^{2} f(w) e^{-2 \varphi(w)} d A(w) .
$$

Lemma 5. Let $f \in L^{\infty} \cap V O$. Then $\tilde{f}(z)-f(z) \rightarrow 0$ as $z \rightarrow \infty$.

Proof. Let $\epsilon>0$. By Theorem 3, (1) and Lemma 4, there is a radius $r \geq 1$ so that

$$
\int_{\mathbb{C} \backslash D^{r}(z)}\left|k_{z}(w)\right|^{2} e^{-2 \varphi(w)} d A(w)<\frac{\epsilon}{\|f\|_{\infty}+1},
$$

for all $z \in \mathbb{C}$. Then

$$
\begin{aligned}
|\tilde{f}(z)-f(z)| & =\left.\left|\int_{\mathbb{C}}(f(w)-f(z))\right| k_{z}(w)\right|^{2} e^{-2 \varphi(w)} d A(w) \mid \\
& \leq \omega_{r}(f)(z)+2\|f\|_{\infty} \int_{\mathbb{C} \backslash D^{r}(z)}\left|k_{z}(w)\right|^{2} e^{-2 \varphi(w)} d A(w) \\
& \leq \omega_{r}(f)(z)+2 \epsilon \leq 3 \epsilon
\end{aligned}
$$

when $|z|$ is large enough. Thus, $\tilde{f}(z)-f(z) \rightarrow 0$ as $|z| \rightarrow \infty$.

For $f \in L^{\infty}$, the Hankel operator $H_{f}$ on $F_{\varphi}^{2}$ with symbol $f$ is defined by

$$
H_{f}(g)=(I-P)(f g) .
$$

Note that $H_{f}$ is a bounded linear operator from $F_{\varphi}^{2}$ into $L_{\varphi}^{2}$ and $\left\|H_{f}\right\| \leq\|f\|_{\infty}$. A useful relationship between Toeplitz and Hankel operators is the well-known multiplication formula

$$
T_{f} T_{g}=T_{f g}-H_{\bar{f}}^{*} H_{g}
$$

which holds for $f, g \in L^{\infty}$. 
In order to characterize compact Hankel operators, we state the definition of functions of vanishing mean oscillation. We say that $f \in L_{\mathrm{loc}}^{2}(\mathbb{C})$ is in $V M O$ if

$$
\lim _{z \rightarrow \infty} M O(f)(z)=0,
$$

where

$$
M O(f)(z)=\left(\frac{1}{A\left(D^{1}(z)\right)} \int_{D^{1}(z)}|f-\hat{f}(z)|^{2} d A\right)^{1 / 2}
$$

and

$$
\hat{f}(z)=\frac{1}{A\left(D^{1}(z)\right)} \int_{D^{1}(z)} f d A
$$

The following two results can be found in [5].

Proposition 6. We have that

$$
V M O=V O+V A
$$

where

$$
V A=\left\{h \in L_{\mathrm{loc}}^{2}(\mathbb{C}): \lim _{z \rightarrow \infty} \widetilde{|h|^{2}}(z)=0\right\}=\left\{h \in L_{\mathrm{loc}}^{2}(\mathbb{C}): \lim _{z \rightarrow \infty} \widehat{|h|^{2}}(z)=0\right\}
$$

Theorem 7. Let $f \in L^{\infty}$. Then $H_{f}$ and $H_{\bar{f}}$ are both compact operators from $F_{\varphi}^{2}$ into $L_{\varphi}^{2}$ if and only if $f \in V M O$.

We finish this section with some basic properties of the Fock spaces $F_{|z|^{\beta}}^{2}$, where $\beta>0$. For any non-negative integer $n$, we define $e_{n}$ by

$$
e_{n}(z)=\frac{z^{n}}{C_{n}}
$$

where

$$
C_{n}^{2}=\left\langle z^{n}, z^{n}\right\rangle_{\beta}=\int_{\mathbb{C}}\left|z^{n}\right|^{2} e^{-|z|^{\beta}} d A(z)=\frac{2 \pi}{\beta} \Gamma\left(\frac{2 n+2}{\beta}\right) .
$$

Then $\left\{e_{n}\right\}_{n \geq 0}$ is an orthonormal basis for $F_{|z|^{\beta}}^{2}$, and it follows that the reproducing kernel function is given by

$$
K_{\beta}(z, w)=\sum_{n=0}^{\infty} \frac{(z \bar{w})^{n}}{C_{n}^{2}}
$$

for $z, w \in \mathbb{C}$, and the orthogonal projection has the following integral representation

$$
\begin{aligned}
P_{\beta} f(z) & =\int_{\mathbb{C}} f(w) K_{\beta}(z, w) e^{-|w|^{\beta}} d A(w) \\
& =\sum_{n=0}^{\infty} \frac{z^{n}}{C_{n}^{2}} \int_{\mathbb{C}} f(w) \bar{w}^{n} e^{-|w|^{\beta}} d A(w) .
\end{aligned}
$$




\section{Proofs of the main Results}

For the proof of the following approximation result, see [2, Lemma 17] and [1, Proposition 9].

Proposition 8. Let $f: \mathbb{C} \rightarrow \mathbb{C}$ be a continuous function in $A$, where $A=L^{\infty}(\mathbb{C}) \cap V O$ or $A=L^{\infty}(\mathbb{C}) \cap V M O$. Then $f$ is bounded away from zero on $\mathbb{C} \backslash D(0, R)$, for some $R>0$, if and only if there is a continuous function $g \in A$ such that $f(z) g(z) \rightarrow 1$ as $z \rightarrow \infty$.

Theorem 9. If $f \in C(\mathbb{C})$ and $f(z) \rightarrow 0$ as $z \rightarrow \infty$, then $T_{f}$ is compact on $F_{\varphi}^{2}$.

Proof. It directly follows from the proof of $(2) \Rightarrow(1)$ in [8, Theorem 5.4].

Proof of Theorem 1. Let $f \in L^{\infty}(\mathbb{C}) \cap V O$ and suppose that there are constants $R>0$ and $m>0$ such that $|f(z)| \geq m$ when $|z|>R$. By Proposition 8 , there exists $g \in L^{\infty}(\mathbb{C}) \cap V O$ such that $f g-1 \rightarrow 0$ as $|z| \rightarrow \infty$. Then

$$
T_{f} T_{g}=I+T_{f g-1}-P M_{f} H_{g}
$$

where $M_{f}$ is the multiplication operator on $L_{\varphi}^{2}$ defined by $M_{f}(h)=f h$. By Theorems 7 and 9 , both $T_{f g-1}$ and $H_{g}$ are compact. Therefore, $T_{f} T_{g}=I+K$ for some compact operator $K$. Similarly, $T_{g} T_{f}=I+K_{1}$, where $K_{1}$ is a compact operator. Thus, $T_{f}$ has a regularizer and hence it is Fredholm.

Conversely, suppose that there are $z_{j} \in \mathbb{C}$ such that $\left|z_{j}\right| \rightarrow \infty$ and $\left|f\left(z_{j}\right)\right| \rightarrow 0$. Then, by Lemma 5. we have that $\widetilde{|f|}\left(z_{j}\right) \rightarrow 0$. Since

$$
\widetilde{|f|^{2}}(z)=\int_{\mathbb{C}}|f(w)|^{2}\left|k_{z}(w)\right|^{2} e^{-2 \varphi(w)} d A(w) \leq\|f\|_{\infty} \widetilde{|f|}(z),
$$

for $z \in \mathbb{C}$, we get that $\widetilde{|f|^{2}}\left(z_{j}\right) \rightarrow 0$. Now (2) gives that

$$
\begin{aligned}
\widetilde{|f|^{2}}\left(z_{j}\right) & =\left\langle T_{|f|^{2}} k_{z_{j}}, k_{z_{j}}\right\rangle=\left\langle\left(T_{\bar{f}} T_{f}+H_{f}^{*} H_{f}\right) k_{z_{j}}, k_{z_{j}}\right\rangle \\
& =\left\langle T_{f} k_{z_{j}}, T_{f} k_{z_{j}}\right\rangle+\left\langle H_{f} k_{z_{j}}, H_{f} k_{z_{j}}\right\rangle=\left\|T_{f} k_{z_{j}}\right\|_{\varphi}^{2}+\left\|H_{f} k_{z_{j}}\right\|_{\varphi}^{2} .
\end{aligned}
$$

Moreover, since $k_{z_{j}} \rightarrow 0$ weakly in $F_{\varphi}^{2}$ and $H_{f}$ is compact on $F_{\varphi}^{2}$ (by Theorem 7), $\left\|H_{f} k_{z_{j}}\right\|_{\varphi}^{2} \rightarrow$ 0 . Therefore, $\left\|T_{f} k_{z_{j}}\right\|_{\varphi}^{2} \rightarrow 0$, and hence $T_{f}$ is not invertible modulo compact operators, or equivalently, $T_{f}$ is not Fredholm.

We now switch our focus and consider Toeplitz operators on $F_{|z|^{\beta}}^{2}$ with $\beta>0$. To prove the index formula of Theorem 2, we consider first Toeplitz operators with symbols of the simple form $(z /|z|)^{m}$.

Lemma 10. For $m \in \mathbb{N}$, let $\psi_{m}(z)=(z /|z|)^{m}$. Then $T_{\psi_{m}}$ is an m-weighted shift operator on $F_{|z|^{\beta}}^{2}$, that is, there are numbers $\alpha_{m, n}>0$ such that $T_{\psi_{m}} e_{n}=\alpha_{m, n} e_{n+m}$ for all $n \geq 0$, where $\left\{e_{n}\right\}_{n \geq 0}$ is defined by (3). Moreover, for any $m \in \mathbb{N}, \alpha_{m, n} \rightarrow 1$ as $n \rightarrow \infty$.

Proof. By (5),

$$
\begin{aligned}
P_{\beta}\left(\psi_{m} e_{n}\right)(z) & =\frac{1}{C_{n}} \sum_{k=0}^{\infty} \frac{z^{k}}{C_{k}^{2}} \int_{\mathbb{C}} \bar{w}^{k} \frac{w^{n+m}}{|w|^{m}} e^{-|w|^{\beta}} d A(w) \\
& =\frac{1}{C_{n}} \frac{z^{n+m}}{C_{n+m}^{2}} \int_{\mathbb{C}}|w|^{2 n+m} e^{-|w|^{\beta}} d A(w)
\end{aligned}
$$


Therefore,

$$
P_{\beta}\left(\psi_{m} e_{n}\right)=\frac{e_{n+m}}{C_{n} C_{n+m}} \int_{\mathbb{C}}|w|^{2 n+m} e^{-|w|^{\beta}} d A(w)=\frac{e_{n+m}}{C_{n} C_{n+m}} \frac{2 \pi}{\beta} \Gamma\left(\frac{2 n+m+2}{\beta}\right),
$$

and so $T_{\psi_{m}} e_{n}=\alpha_{m, n} e_{n+m}$ with

$$
\alpha_{m, n}=\frac{1}{C_{n} C_{n+m}} \frac{2 \pi}{\beta} \Gamma\left(\frac{2 n+m+2}{\beta}\right) .
$$

Then, by (4), Stirling's formula shows that, for any $m, \alpha_{m, n} \rightarrow 1$ as $n \rightarrow \infty$.

Proposition 11. For every $m \in \mathbb{N}$, the Toeplitz operator $T_{\bar{\psi}_{m}}$ is a Fredholm operator on $F_{|z|^{\beta}}^{2}$ of index $m$.

Proof. Let

$$
f(z)=\sum_{n=0}^{\infty} \lambda_{n} e_{n}
$$

be a function in $F_{|z|^{\beta}}^{2}$. By Lemma 10 ,

$$
T_{\psi_{m}} f=\sum_{n=0}^{\infty} \lambda_{n} T_{\psi_{m}} e_{n}=\sum_{n=0}^{\infty} \lambda_{n} \alpha_{m, n} e_{n+m}
$$

where $\alpha_{m, n} \neq 0$, for any $n \geq 0$, and $\alpha_{m, n} \rightarrow 1$ as $n \rightarrow \infty$. It follows that $\operatorname{dim} \operatorname{ker} T_{\psi_{m}}=0$ and $\operatorname{dim}\left(F_{|z|^{\beta}}^{2} / T_{\psi_{m}}\left(F_{|z|^{\beta}}^{2}\right)\right)=m$. Therefore, $T_{\psi_{m}}$ is a Fredholm operator of index $-m$, and hence $T_{\bar{\psi}_{m}}=T_{\psi_{m}}^{*}$ is Fredholm of index $m$.

We can now prove the index formula of Toeplitz operators on $F_{|z|^{\beta}}^{2}$ with symbols in the class $L^{\infty} \cap V O$.

Proof of Theorem 2. Taking into account Proposition 8 and its proof, Theorem 9, (2), and Proposition 11, this proof is mutatis mutandis the proof of [1, Theorem 20].

\section{REFERENCES}

[1] A. Al-Qabani and J. A. Virtanen. Fredholm theory of Toeplitz operators on standard weighted Fock spaces. Ann. Acad. Sci. Fenn. Math., 43(2):769-783, 2018.

[2] C. A. Berger and L. A. Coburn. Toeplitz operators on the Segal-Bargmann space. Trans. Amer. Math. Soc., 301(2):813-829, 1987.

[3] H. R. Cho and K. Zhu. Fock-Sobolev spaces and their Carleson measures. J. Funct. Anal., 263(8):24832506, 2012.

[4] R. Fulsche and R. Hagger. Fredholmness of Toeplitz Operators on the Fock Space. Complex Anal. Oper. Theory, 13(2):375-403, 2019.

[5] Z. Hu and X. Lv. Hankel operators on weighted Fock spaces (in Chinese). Sci. China Math., 46(2):141$156,2016$.

[6] N. Marco, X. Massaneda, and J. Ortega-Cerdà. Interpolating and sampling sequences for entire functions. Geom. Funct. Anal., 13(4):862-914, 2003.

[7] J. Marzo and J. Ortega-Cerdà. Pointwise estimates for the Bergman kernel of the weighted Fock space. J. Geom. Anal., 19(4):890-910, 2009.

[8] R. Oliver and D. Pascuas. Toeplitz operators on doubling Fock spaces. J. Math. Anal. Appl., 435(2):1426-1457, 2016.

[9] G. Schneider. Hankel operators with antiholomorphic symbols on the Fock space. Proc. Amer. Math. Soc., 132(8):2399-2409, 2004. 
[10] G. Schneider and K. A. Schneider. Generalized Hankel operators on the Fock space. Math. Nachr., 282(12):1811-1826, 2009.

[11] A. P. Schuster and D. Varolin. Toeplitz operators and Carleson measures on generalized Bargmann-Fock spaces. Integral Equations Operator Theory, 72(3):363-392, 2012.

[12] E. M. Stein. Harmonic analysis: real-variable methods, orthogonality, and oscillatory integrals. Princeton Mathematical Series, 43. Monographs in Harmonic Analysis, III. Princeton University Press, Princeton, NJ, 1993.

[13] K. Stroethoff. Hankel and Toeplitz operators on the Fock space. Michigan Math. J., 39(1):3-16, 1992.

[14] K. Zhu. Analysis on Fock spaces. Graduate Texts in Mathematics, 263. Springer, New York, 2012.

Department of Mathematics and Computer Applications, Al-Nahrain University, BaghDAD, IRAQ

E-mail address: aminarasim@hotmail.com

Department of Mathematics, University of Reading, Whiteknights, Reading RG6 6AX, ENGLAND

E-mail address: t.w.hilberdink@reading.ac.uk

E-mail address: j.a.virtanen@reading.ac.uk 